\title{
Recent Applications of Polyethylene Glycols (PEGs) and PEG Derivatives
}

\author{
Daniela Hutanu ${ }^{1 \#}$, Mark D Frishberg ${ }^{1}$, Lihong Guo ${ }^{1}$ and Costel C Darie ${ }^{2 *}$
}

${ }^{1}$ JenKem Technology USA, Plano, TX-75024, USA

${ }^{2}$ Clarkson University, Potsdam, NY-13699, USA

\#This author also equally contributed

\begin{abstract}
Numerous applications of polyethylene glycol derived polymers (PEGs) have been reported in the scientific literature for many years. With increasing experience and comfort by regulatory authorities, worldwide, in the utilization of these materials in drug and medical device applications, their use in a variety of research and development areas is expanding. This review will focus on just the range of applications of PEGs published in the first half of 2014 in the medical device, drug development, and diagnostics areas, including drug delivery, wound healing, cell culture models, and tissue regeneration.
\end{abstract}

Keywords: Polyethylene glycols; Drug development; Drug-release

\section{Introduction}

Polyethylene glycols (PEGs) are hydrophilic oligomers or polymers synthesized from ethylene oxide, consisting of a repeating unit of - $(\mathrm{O}$ $\left.-\mathrm{CH}_{2}-\mathrm{CH}_{2}\right)$-. PEGs are synthesized in a wide range of molecular weights, referred to as "monodispersed", when there is a defined PEG chain size and molecular weight, or more commonly as "polydispersed" polymers, when there is a Gaussian distribution of chain lengths and molecular weights. The ability to attach a variety of reactive functional groups to the terminal sites of PEG polymers has greatly expanded their benefits. Hetero- and homo-bifunctional PEG derivatives are especially suitable as cross-linking agents or spacers between two chemical entities [1-3], whereas mono-functional PEGs prevent bridging reactions which may otherwise affect the PEGylation of certain compounds with bifunctional PEGs [4-7]. The sterically bulky structure of branched PEGs consisting of linear methoxy PEG chains attached to a central core, i.e., Y-shaped branched PEG derivatives, facilitates the single point attachment to targets $[8,9]$ via a single reactive group. Multiarm PEG derivatives (Figure 1) prepared by ethoxylation of different cores such as pentaerythritol, hexaglycerol, or tripentaerythritol, and incorporation of reactive moieties, are cross-linked easily into threedimensional hydrogel materials [10-14] (Figures 1a-1c).
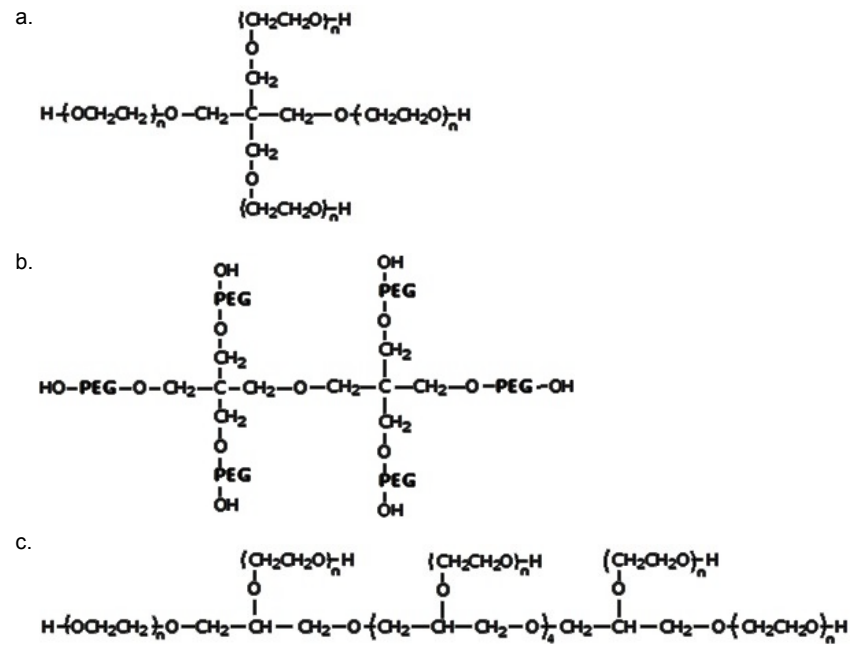

Figure 1: Representative illustrations of molecular structures for multiarm underivatized polyethylene glycols

a. Molecular structure of an underivatized 4 arm polyethylene glycol

b. Molecular structure of an underivatized 6arm polyethylene glycol polymer. In this particular structure, "PEG" stands for $-\left(\mathrm{CH}_{2} \mathrm{CH}_{2} \mathrm{O}\right)_{n}-$

c. Molecular structure of an underivatized 8arm polyethylene glycol polymer
The process of PEGylation, the covalent grafting of a PEG derivative onto molecules, improves the water solubility and biocompatibility, especially useful for drug development [15]. PEGylated products require extensive characterization with complex analytical techniques [16-18] to ensure regulatory compliance for medical applications. Bifunctional PEG derivatives are used frequently for the PEGylation of peptides [19-21], proteins [22,23], small molecules like folate, mannose, prodrugs [3,24], oligonucleotides [25], cells [26], nanoparticles and virus particles [23,27-32], and surfaces [33-37]. Multi-arm PEG derivatives are mostly employed in the formation of hydrogels [14,19,38-56] for controlled release of therapeutics; for use in medical devices; regenerative medicine; and in various other applications, including cell culture, wound sealing, and wound healing. Significant advances were made in 2014 by the scientific community in the research and development of novel applications for PEGs. Table 1 summarizes select novel applications of PEGs described in scientific publications in the first half of 2014, while the remainder of the publication highlights just those 2014 PEG applications related to the medical field.

\section{Applications of Polyethylene Glycols for Cancer Drug Delivery and Targeted Diagnostics}

R\&D efforts on novel applications for PEG derivatives, published in the first half of 2014, focus in majority on drug delivery and targeted diagnostics, either via direct PEGylation of therapeutics [2,3,21,6265]; or via PEG-containing vehicles, such as nanoparticles [64,65], liposomes [59], dendrimers [32,60], or micelles [57,58,61]. Important parameters that influence the bioactivity of the PEGylated drugs include the length of the PEG chain, the PEGylation site, the linker chemistry, and the temperature selected for the PEGylation reaction. For example, heat treatment was shown to improve the bioactivity of C-terminally PEGylated staphylokinases, whereas an amyl linker for a $20 \mathrm{kDa}$ PEG increased significantly the bioactivity of staphylokinases [62]. The PEGylation of proteins is greatly influenced by the solvent

*Corresponding author: Costel C Darie, Biochemistry and Proteomics Group, Department of Chemistry and Biomolecular Science, Clarkson University, 8 Clarkson Avenue, Potsdam, NY 13699-5810, USA, Tel: 315-268-7763; Fax: 315 268-6610; E-mail: cdarie@clarkson.edu

Received July 21, 2014; Accepted August 22, 2014; Published August 26, 2014

Citation: Hutanu D, Frishberg MD, Guo L, Darie CC (2014) Recent Applications of Polyethylene Glycols (PEGs) and PEG Derivatives. Mod Chem appl 2: 132. doi:10.4172/2329-6798.1000132

Copyright: $\odot 2014$ Hutanu D, et al. This is an open-access article distributed under the terms of the Creative Commons Attribution License, which permits unrestricted use, distribution, and reproduction in any medium, provided the original author and source are credited. 
Citation: Hutanu D, Frishberg MD, Guo L, Darie CC (2014) Recent Applications of Polyethylene Glycols (PEGs) and PEG Derivatives. Mod Chem appl 2: 132. doi:10.4172/2329-6798.1000132

Page 2 of 6

\begin{tabular}{|c|c|c|}
\hline Polyethylene Glycol (PEG) Compound & PEG Applications & Reference \\
\hline Biotin PEG SGA Ester, MW 3500 & Cell PEGylation & [26] \\
\hline Methoxy PEG Amine, HCI Salt, MW 2000 & Diagnostics & [57] \\
\hline 4arm PEG Succinimidyl Carboxymethyl Ester, MW 40000 & Drug delivery & [24] \\
\hline Maleimide PEG Amine, MW 3500 & Drug delivery & [2] \\
\hline Maleimide PEG Hydroxyl, MW 3500 & Drug delivery & [58] \\
\hline Maleimide PEG NHS Ester MW 1000 & Drug delivery & [59] \\
\hline Maleimide PEG NHS Ester, MW 2000 & Drug delivery & [21] \\
\hline Maleimide PEG NHS Ester, MW 3500 & Drug delivery & [59] \\
\hline Maleimide PEG NHS Ester, MW 5000 & Drug delivery & [60] \\
\hline Methoxy PEG Amine, MW 12kDa & Drug delivery & [61] \\
\hline Methoxy PEG Amine, MW 20kDa & Drug delivery & [62] \\
\hline Methoxy PEG Carboxyl MW 3500 & Drug delivery & [32] \\
\hline Methoxy PEG Carboxyl, MW 1000 & Drug delivery & [63] \\
\hline Methoxy PEG NHS Ester, MW 5000 & Drug delivery & [60] \\
\hline Methoxy PEG Propionaldehyde MW 5000, MW 20kDa & Drug delivery & [62] \\
\hline PEG Maleimide, MW 10000 & Drug delivery & [22] \\
\hline PEG NHS Ester MW 5000 & Drug delivery & [59] \\
\hline PEG Succinimidyl Carbonate, MW 10000 & Drug delivery & [22] \\
\hline PEG Thiol, MW 5000 & Drug delivery & [59] \\
\hline Amine PEG Carboxyl, HCI Salt, MW 5000 & Drug delivery, Diagnostics & [3] \\
\hline t-Boc Amine PEG Amine, HCI Salt, MW 5000 & Drug delivery, Diagnostics & [3] \\
\hline Amine PEG Carboxyl MW 3500 & Drug delivery, Nanoparticle PEGylation & [64] \\
\hline Methoxy PEG Amine MW 3500 & Drug delivery, Nanoparticle PEGylation & [64] \\
\hline 4arm PEG Succinimidyl Carboxymethyl Ester, MW 10000 & Hydrogel & [55] \\
\hline 4arm PEG Amine, MW 20kDa & Hydrogel & {$[14],[50],[53]$} \\
\hline 4arm PEG Thiol MW 10kDa & Hydrogel & [41] \\
\hline 4arm Polyethylene Glycol MW 10kDa & Hydrogel & [41] \\
\hline 4arm Polyethylene Glycol MW 20kDa & Hydrogel & [14], [43],[40], [45], [46], [50] \\
\hline 4arm Polyethylene Glycol MW 40kDa & Hydrogel & [46] \\
\hline 4arm Polyethylene Glycol, MW 5000 & Hydrogel & [56] \\
\hline 8arm PEG Amine (tripentaerythritol), HCI Salt, MW 40000 & Hydrogel & [39] \\
\hline 8arm PEG Amine HCl Salt MW 10kDa & Hydrogel & [47] \\
\hline 8arm PEG Thiol (hexaglycerol), MW 10000 & Hydrogel & [44] \\
\hline 8arm Polyethylene Glycol (hexaglycerol), MW 10000 & Hydrogel & [44], [53] \\
\hline 8arm Polyethylene Glycol (hexaglycerol), MW 15000 & Hydrogel & [49] \\
\hline 8arm Polyethylene Glycol (hexaglycerol), MW 20000, MW 40000 & Hydrogel & [38] \\
\hline Acrylate PEG NHS Ester MW 3500 & Hydrogel & [42], [48] \\
\hline Methoxy PEG Amine, MW 2000 & Hydrogel & [54] \\
\hline 6arm PEG Amine MW 10kDa & Nanoparticle PEGylation & [29] \\
\hline Methoxy PEG Amine, MW 10000 & Nanoparticle PEGylation & [65] \\
\hline PEG Amine, MW 2000 & Nanoparticle PEGylation & [29] \\
\hline PEG NHS Ester MW 10kDa, MW 20kDa, MW 40kDa & Nanoparticle PEGylation & [27] \\
\hline Thiol PEG Amine, MW 5000 & Nanoparticle PEGylation & [28] \\
\hline Methoxy PEG Amine, MW 5000 & Nanoparticle PEGylation, Drug delivery & [30], [62] \\
\hline Methoxy Polyethylene Glycol, MW 5000 & Nanoparticle PEGylation, Drug delivery & [30] \\
\hline 8arm Polyethylene Glycol (tripentaerythritol), MW 20000 & Peptide PEGylation & [19] \\
\hline Methoxy PEG Amine HCI Salt MW 5000 & Peptide PEGylation & [20] \\
\hline Biotin PEG NHS Ester & Protein PEGylation & [35] \\
\hline Methoxy PEG NHS Ester & Protein PEGylation & [35] \\
\hline Thiol PEG Carboxyl, MW 5000 & Protein PEGylation, Nanoparticle PEGylation & [23] \\
\hline PEG Amine, HCl Salt, MW 20000 & Surface modification & [33] \\
\hline PLL20k-G35-PEG2k & Surface modification & {$[34,36,37]$} \\
\hline
\end{tabular}

Table 1: Select PEG compounds and their applications, cited in the first half of 2014.

used during the conjugation to the PEG. Peng et al. [22] discovered that organic solvents such as DMSO increase the degree of PEGylation, minimize the PEG hydrolysis, and decrease the PEGylation time for hydrophobic proteins such as G-CSF, compared to PEGylation in water phase. Selecting an organic solvent for hydrophobic proteins has the potential to reduce the cost of the reagents and the reaction times, parameters important for PEGylation processes on an industrial scale.
Among the many improvements brought to therapeutics by PEGylation are the increased water solubility, improved stability, controlled release, extended drug half-life, and an enhanced PK/ PD (pharmacokinetic/pharmacodynamic) profile. PEGylation of therapeutic proteins occurs mainly on the N-terminal group, carbohydrates, sulfhydryl, and the aminoacids Thr, Cys, Asp, Glu Lys, His, Arg, Tyr, and Ser. In the course of the PEGylation of small 
molecule drugs, a multi-arm polyethylene glycol will bond multiple drug molecules, ensuring a high drug load and an enhanced drugrelease function [15]. As an example, increasing the molecular weight of the iRGD peptide by PEGylation prolonged the macromolecular extravasation and the overall drug penetration into tumors, and improved the pharmacokinetic profile of iRGD as compared to the unmodified peptide [21]

PEG-containing vehicles for drug delivery such as liposomes [59], dendrimers [60], nanoparticles [64,65], or micelles $[57,58,61]$ are valid alternatives to direct PEGylation of drugs. Mei et al. [59] developed a multistage liposome drug delivery system co-modified with RGD, TAT, a specific ligand and a penetrating peptide, containing a cleavable PEG that increased the stability and circulation time of the liposomes. Liposomes undergo passive extravasation to tumor tissues, where the dual ligands become exposed through controlled exogenous administration of reducing l-cysteine. Subsequently, RGD recognize integrins, commonly overexpressed on malignant tumors, and mediate the internalization in a synergistic effect with TAT, penetrating deep into avascular tumor spheroids. Another type of PEG containing vehicle, a multifunctional dendrimeric carrier was developed by Kong et al. [60] for targeted delivery of the hydrophobic anticancer drug 10-hydroxycamptothecin (10-HCPT). The dendrimers consisted of integrated hydrophobic C12 alkyl chains with polyethylene glycol chains, and $c$ (RGDfK) ligands on the surface. The dendrimer-10HCPT drug complex exhibited higher drug loading and stability, and increased water solubility when compared to free 10-HCPT drug. The dendrimer-drug complex showed a higher cytotoxicity towards 22RV1 cells that overexpress integrin $\alpha v \beta 3$, and a lower cytotoxicity against MCF-7 cells with lower levels of integrin $\alpha v \beta 3$, following selective internalization of the complex into carcinoma cells via integrin receptor mediated endocytosis.

Drug delivery via PEG-modified nanoparticles is described in several 2014 publications. Super paramagnetic iron oxide nanoparticles containing a self-assembled copolymer of reducible polyamidoamine (rPAA) with polyethylene glycol/dodecyl amine on the surface, were employed for Doxorubicin delivery for cancer therapy [30]. For drug delivery, the intercalating area between the alkyl grafts of reducible copolymers and the oleic acid layer on the surface of the nanoparticles stored the hydrophobic drug, while the PEG chains improved the dispersion of the nanoparticles in aqueous environment. The Doxorubicin delivered via these nanoparticles inhibited successfully the growth of xenograft MDA-MB-231 breast tumors in mice. Drug encapsulation in maleimide-polyethyleneglycol- poly(d,l-lactic-coglycolide) particles (PEG-PLGA) was investigated for targeted drug delivery of Cisplatin [2]. The Cisplatin encapsulating particles were produced using a single step electrospray technique, and were further modified with a CD44 monoclonal antibody targeting the counterpart receptor. Cisplatin-encapsulating CD44-PEG-PLGA particles targeted efficiently CD44-overexpressed ovarian cancer cells, and exhibited an increased anti-proliferative ability at normal chemotherapy concentrations, as compared to the free form of Cisplatin. Polyethyleneglycol-poly (l-lactic-co-glycolic acid) nanoparticles were also employed for targeted drug delivery of Paclitaxel [64]. PEG-PLGA nanoparticles functionalized with an iNGR moiety presented the highest accumulation and deepest penetration at glioma sites. Paclitaxel-loaded iNGR-NP showed promising anti-angiogenesis activity and improved survival time for mice with intracranial glioma.

Micellar drug delivery systems were explored by Xu et al. [63] for delivery of atorvastatin calcium (Ator). Delivery micelles consisted of amphiphilic copolymers of methoxy polyethylene glycol-s-s-vitamin
E succinate (PSV). Ator-loaded PSV micelles showed good colloidal stability, high drug loading, and great encapsulation efficiency. The release of the Ator drug into the cytosol was facilitated by detachment of the PEG shell in the presence of high concentration of intracellular glutathione. The Ator-loaded micelles were shown to inhibit significantly the migration and invasion of $4 \mathrm{~T} 1$ metastatic breast cancer cells. Dual drug delivery coupled with a targeted approach in a polypeptide-based micelle system was accomplished by Song et al. [58]. The micelle was composed of an amphiphilic copolymer prepared by grafting $\alpha$-tocopherol and polyethylene glycol onto poly(lglutamic acid), while the surface of the micelles was modified with an av $\beta 3$ integrin targeting peptide, $c$ (RGDfK). The incorporation into micelles of two drugs, Docetaxel and Cisplatin, was accomplished via hydrophobic and chelation effects. The drugs co-delivered in micelles showed synergistic cytotoxicity, long circulation time, and enhanced internalization into mouse melanoma (B16F1) cells. Polymeric micelles have also been employed for diagnostic purposes as delivery vehicles for diagnostic reagents. As a first example, Kim et al. [57] developed $\mathrm{pH}$-responsive polymeric micelles loaded with MRI contrast agents for use in cancer diagnostics. Self-assembled micelles made of amphiphilic block copolymers: methoxy polyethylene glycol-b-poly(lhistidine) and methoxy polyethylene glycol-b-poly(l-lactic acid)diethylenetriaminopentaacetic acid dianhydride-gadolinium chelate, proved stable at physiological $\mathrm{pH}$, but collapsible in acidic conditions due to protonation of imidazole groups. The destabilization of the micelles in the acidic tumoral environment allowed the preferential accumulation of the MRI contrast agent in the tumoral regions, enabling the detection of small tumors within minutes. As a second example, Guo et al. [61] reported the development of PEG-polyaspartamide micelles loaded with a photosensitizer (Ce6) and cyanine dye (Cypate), with a dual role, cancer diagnostics and cancer photo-therapy. Photosensitizerloaded micelles that also integrated a cyanine dye enabled localizing of the tumors via dual photoacoustic/near-infrared fluorescent imaging, and simultaneously induced photothermal damage to cancer cells by sequential synergistic photothermal /photodynamic therapy.

\section{Applications of Polyethylene Glycol Hydrogels in Wound Healing and Tissue Regeneration}

Another major reported use of polyethylene glycols in 2014 referenced papers is for the development of hydrogels [14,19,38-56]. Among the common uses of PEG hydrogels are the use as adhesives for wound closure, as controlled release matrices for therapeutics, for wound healing, as part of medical devices, and as regenerative medicine tools. A biodegradable cytocompatible bioadhesive hydrogel system was developed by Jeon et al. [47] from on oxidized methacrylated alginate/8-arm polyethylene glycol amine used for culture of human bone marrow-derived mesenchymal stem cells. The swelling behavior, degradation profiles, and storage moduli of the hydrogel bioadhesive were adjusted by varying the degree of oxidation of the alginate. The adhesion level was enhanced on a porcine skin model as compared to commercially available fibrin glue. Another bio-adhesive hydrogel, Catechol-containing 4-armed PEG end-capped with dopamine, was researched by Cencer et al. [55]. Studies on the effect of $\mathrm{pH}$ on the rate of intermolecular cross-linking and adhesion to biological substrates allowed the identification of the optimal buffering $\mathrm{pH}$ for formulation of the adhesive. When tested as an adhesive for pericardium tissues, a formulation $\mathrm{pH}$ of 7.4 provided the ideal balance of curing rate, mechanical properties, and interfacial binding ability for the Catecholcontaining 4-armed PEG hydrogel end-capped with dopamine.

Progress on research and development of regenerative therapies aided by PEG hydrogel matrices is reported in several 2014 publications. 
As an example, a study by Frith et al. [39] describes a combination of mesenchymal progenitor cells with a potentially injectable PEG/ hyaluronic acid degradable hydrogel with a covalently bound pentosan polysulphate, that showed promise as therapy for the regeneration of the cartilage tissue. The molecular weight of the polyethylene glycol chains does not have a significant effect on cell division and sulfated glycosaminoglycan production as part of composite hydrogels [46]. Likewise, for regenerative medicine purposes, Hoffman et al. [48] created a "tissue engineered periosteum", utilizing hydrolytically degradable PEG hydrogels to transplant and localize mesenchymal stem cells to allograft surfaces. The approach increased the autograph healing-graft vascularization, the endochondral bone formation, and the biomechanical strength, when compared to untreated allografts. As a drawback, the process of endochondral ossification was delayed compared to untreated allografts, requiring future supplementation of the hydrogels with additives to expedite the ossification process for treatment of critical sized bone defects. Nguyen et al. [44] controlled the in situ osteogenic differentiation of encapsulated stem cells in polyethylene glycol hydrogels by employing localized, and prolonged delivery of siRNA and/or miRNA interfering molecules. Moreover, differentiation of encapsulated human mesenchymal stem cells down the osteogenic lineage was achieved by Hao et al. [14] by in-situ cell culture in a 4 arm PEG hydrogel scaffold, by varying the rate and type of the hydrogel degradation. A 4arm PEG hydrogel was synthesized via a mixed-mode step-and-chain-growth photo-polymerization in visible light, using functionalized 4-arm polyethylene glycol as a backbone macromer, eosin-Y as a photosensitizer, a di-thiol containing molecule as co-initiator/cross-linker, and N-vinylpyrrolidone (NVP) as a gelation accelerator. The gelation kinetics, gel degradation, swelling and stiffness, were dependent on the identity of the functional groups, the identity of the cross-linkers, as well as on the concentration of the cross-linker and NVP.

\section{Applications of Polyethylene Glycol Hydrogels in Cell Culture and Tissue Models}

PEG and PEG-copolymer hydrogels are practical solutions as scaffolds and have been used for cell culture; for controlled release of therapeutics; and for various other applications, including but not limited to tissue engineering. PEG hydrogels with physiologically relevant matrix elasticities and diffusion distance, fabricated in transwell inserts, were utilized by Gould et al. [38] for cell culture of valvular interstitial cells and valvular endothelial cells. Growth of the cells in the PEG hydrogel matrices has enabled relevant investigations related to aortic valve stenosis initiation and progression. As a replacement to the traditional 2D cell culture techniques for human Pluripotent Stem Cells (hPSCs), Dang et al. [42] proposed polyethylene glycol hydrogels as cell growth scaffolds, with the addition of an immobilized soluble angiopoeitin-1-derived peptide, QHREDGS. The addition of the peptide enabled a dose-dependent increase in the cell adhesion into a well-defined network, simultaneously inhibiting the apoptosis of hPSCs. The morphology of the human bone marrow stromal cells was demonstrated by Farooque et al. [40] to be closer to physiological conditions in 3D PEG tetramethacrylate hydrogels scaffolds, compared to traditional 2D planar conditions. In another application, Daniele et al. [41] developed a bio-synthetic tissue scaffold consisting of an interpenetrating network of gelatin methacrylamide polymerized within a polyethylene glycol framework for the culture of endothelial cells. This tissue model showed extensive cytoplasmic spreading and cellular adhesion, with sustained viability and proliferation for encapsulated and adherent cells.
Lin et al. [45] developed an orthogonal thiol-ene PEG hydrogel system for cell culture of liver cell lines, Huh7 and HepG2. The hydrogel system was based on a poly(ethylene glycol)-tetra-norbornene macromere, which allowed facile incorporation of bioactive peptides (e.g., fibronectin-derived RGDS) for improved cell-matrix interactions,. According to the authors, this $3 \mathrm{D}$ hydrogel system is the first to upregulate the expression of NCTP in encapsulated Huh7 and HepG2 cell lines and the hepatocyte-like polarity, without genetic modification, and without the need for growth factors and chemical additives. The effect of substrate stiffness on the responsiveness of melanoma cells to treatment with a pharmacological inhibitor, PLX4032, was studied by Tokuda [43] on cell cultures on PEG hydrogel. The use of PEG hydrogels revealed stage-dependent responses to PLX4032 that were not detectable in traditional culturing techniques. For example, the researchers have demonstrated that A375 cells' response to treatment does not depend on the stiffness of the tissue; whereas WM35 cells are more dependent on the substrate modulus, displaying increased apoptosis and decreased focal adhesions on the substrate. Zhang [49] determined that larger amounts of ammonia cross linker used in the polymerization of an 8 arm PEG macromere, lead to higher crosslinking density and bulk of hydrogels, an increased surface elasticity and, generally, to smoother surface morphologies. The amount of cross linker is therefore important in the polymerization of PEG-based hydrogels formed by amine-Michael type addition.

\section{Conclusions}

Several noteworthy research papers published in the first half of 2014 were reviewed in detail in this publication, describing the use of polyethylene glycols and PEG hydrogels in cancer diagnostics and drug delivery, wound healing, tissue regeneration, cell culture, and as tissue scaffold models. Other applications of polyethylene glycols reported in early 2014 are summarized briefly in Table 1, including for PEGylation of small and large molecules, such as peptides, proteins, folate, mannose, oligonucleotides; PEGylation of cells, nanoparticles and virus particles; and for surface modification.

\section{References}

1. Zhou J, Hao G, Weng H, Tsai YT, Baker DW, et al. (2013) In vivo evaluation of medical device-associated inflammation using a macrophage-specific positron emission tomography (PET) imaging probe. Bioorg Med Chem Lett 23: 20442047.

2. Bai MY, Liu SZ (2014) A simple and general method for preparing antibodyPEG-PLGA sub-micron particles using electrospray technique: an in vitro study of targeted delivery of cisplatin to ovarian cancer cells. Colloids Surf B Biointerfaces 117: 346-353

3. Baker DW, Zhou J, Tsai YT, Patty KM, Weng H, et al. (2014) Development of optical probes for in vivo imaging of polarized macrophages during foreign body reactions. Acta Biomater 10: 2945-2955.

4. Jin M, Chen W, Huang W, Rong L, Gao Z (2013) Preparation of pegylated lumbrokinase and an evaluation of its thrombolytic activity both in vitro and in vivo. Acta Pharmaceutica Sinica B 3: 123-129.

5. Proulx ST, Luciani P, Christiansen A, Karaman S, Blum KS, et al. (2013) Use of a PEG-conjugated bright near-infrared dye for functional imaging of rerouting of tumor lymphatic drainage after sentinel lymph node metastasis. Biomaterials 34: 5128-5137.

6. Hou B, Li S, Li X, Xiu Z (2007) Design, Preparation and in vitro Bioactivity of Mono-PEGylated Recombinant Hirudin. Chinese Journal of Chemical Engineering 15: 775-780.

7. Sun G, Lin X, Shen L, Wu F, Xu D, et al. (2013) Mono-PEGylated radix ophiopogonis polysaccharide for the treatment of myocardial ischemia. Eur $\mathrm{J}$ Pharm Sci 49: 629-636.

8. Resch G, Moreillon P, Fischetti VA(2011) PEGylating a bacteriophage endolysin inhibits its bactericidal activity. AMB Express 1: 29. 
9. Hoehlig K, Maasch C, Shushakova N, Buchner K, Huber-Lang M, et al. (2013) A novel C5a-neutralizing mirror-image (I-)aptamer prevents organ failure and improves survival in experimental sepsis. Mol Ther 21: 2236-2246.

10. Van de Manakker F, van der Pot M, Vermonden T, van Nostrum CF, Hennink WE (2008) Self-Assembling Hydrogels Based on $\beta$-Cyclodextrin/Cholesterol Inclusion Complexes. Macromolecules 41: 1766-1773.

11. van Dijk M, van Nostrum CF, Hennink WE, Rijkers DT, Liskamp RM (2010) Synthesis and characterization of enzymatically biodegradable PEG and peptide-based hydrogels prepared by click chemistry. Biomacromolecules 11 : 1608-1614.

12. Maitz MF, Freudenberg U, Tsurkan MV, Fischer M, Beyrich T, et al. (2013) Bioresponsive polymer hydrogels homeostatically regulate blood coagulation. Nat Commun 4: 2168.

13. Tong X, Yang F (2014) Engineering interpenetrating network hydrogels as biomimetic cell niche with independently tunable biochemical and mechanical properties. Biomaterials 35: 1807-1815.

14. Hao Y, Shih H, Munoz Z, Kemp A, Lin CC (2014) Visible light cured thiol-viny hydrogels with tunable degradation for $3 D$ cell culture. Acta Biomater 10: 104114.

15. Veronese FM, Pasut G (2005) PEGylation, successful approach to drug delivery. Drug Discov Today 10: 1451-1458.

16. Myers BK, Zhang B, Lapucha JE, Grayson SM (2014) The characterization of dendronized poly(ethylene glycol)s and poly(ethylene glycol) multi-arm stars using matrix-assisted laser desorption/ionization time-of-flight mass spectrometry. Anal Chim Acta 808: 175-189.

17. Zhu M, Carta G (2014) Adsorption of polyethylene-glycolated bovine serum albumin on macroporous and polymer-grafted anion exchangers. J Chromatogr A 1326: 29-38

18. Hutanu D, Darie CC (2014) Trends in Characterization of PEGylated Proteins by Mass Spectrometry. Mod Chem Appl.

19. Nguyen EH, Zanotelli MR, Schwartz MP, Murphy WL (2014) Differentia effects of cell adhesion, modulus and VEGFR-2 inhibition on capillary network formation in synthetic hydrogel arrays. Biomaterials 35: 2149-2161.

20. Moreno M, Zurita E, Giralt E (2014) Delivering wasp venom for cancer therapy J Control Release 182: 13-21.

21. Pang HB, Braun GB, She ZG, Kotamraju VR, Sugahara KN, et al. (2014) A free cysteine prolongs the half-life of a homing peptide and improves its tumorpenetrating activity. J Control Release 175: 48-53.

22. Peng F, Liu Y, Li X, Sun L, Zhao D, et al. (2014) PEGylation of G-CSF in organic solvent markedly increase the efficacy and reactivity through protein unfolding, hydrolysis inhibition and solvent effect. J Biotechnol 170: 42-49.

23. Tian L, Tadepalli S, Hyun Park S, Liu KK, Morrissey JJ, et al. (2014) Bioplasmonic calligraphy for multiplexed label-free biodetection. Biosens Bioelectron 59: 208-215.

24. Santi DV, Schneider EL, Ashley GW (2014) Macromolecular prodrug that provides the irinotecan (CPT-11) active-metabolite SN-38 with ultralong halflife, low $\mathrm{C}(\max )$, and low glucuronide formation. J Med Chem 57: 2303-2314.

25. An S, Kuang Y, Shen T, Li J, Ma H, et al. (2013) Brain-targeting delivery for RNA neuroprotection against cerebral ischemia reperfusion injury. Biomaterials 34 : 8949-8959.

26. Liu B, Chen W, Evavold BD, Zhu C (2014) Accumulation of dynamic catch bonds between TCR and agonist peptide-MHC triggers $\mathrm{T}$ cell signaling. Cell 157: 357-368.

27. Saville SL, Qi B, Baker J, Stone R, Camley RE, et al. (2014) The formation of linear aggregates in magnetic hyperthermia: implications on specific absorption rate and magnetic anisotropy. J Colloid Interface Sci 424: 141-151.

28. Zhang Z, Li W, Zhao Q, Cheng M, Xu L, et al. (2014) Highly sensitive visual detection of copper (II) using water-soluble azide-functionalized gold nanoparticles and silver enhancement. Biosens Bioelectron 59: 40-44.

29. Chong $Y$, Ma $Y$, Shen $H$, Tu X, Zhou X, et al. (2014) The in vitro and in vivo toxicity of graphene quantum dots. Biomaterials 35: 5041-5048.

30. Chen J, Shi M, Liu P, Ko A, Zhong W, et al. (2014) Reducible polyamidoaminemagnetic iron oxide self-assembled nanoparticles for doxorubicin delivery. Biomaterials 35: 1240-1248.
31. Tesfay MZ, Kirk AC, Hadac EM, Griesmann GE, Federspiel MJ, et al. (2013) PEGylation of vesicular stomatitis virus extends virus persistence in blood circulation of passively immunized mice. J Virol 87: 3752-3759.

32. Gajbhiye V, Escalante L, Chen G, Laperle A, Zheng Q, et al. (2014) Drugloaded nanoparticles induce gene expression in human pluripotent stem cell derivatives. Nanoscale 6: 521-531.

33. Miller DJ, Kasemset S, Wang L, Paul DR, Freeman BD (2014) Constant flux crossflow filtration evaluation of surface-modified fouling-resistant membranes. Journal of Membrane Science 452: 171-183.

34. Portran D (2014) Micropatterning Microtubules. Methods in Cell Biology. Matthieu P, Manuel T (Editors) Academic Press, USA. 39-51.

35. Petek NA, Mullins RD (2014) Bacterial actin-like proteins: purification and characterization of self-assembly properties. Methods Enzymol 540: 19-34.

36. Boujemaa-Paterski R, Galland R, Suarez C, Guérin C, Théry M, et al. (2014) Directed actin assembly and motility. Methods Enzymol 540: 283-300.

37. Vignaud T, Ennomani H, Théry M (2014) Polyacrylamide hydroge micropatterning. Methods Cell Biol 120: 93-116.

38. Gould ST, Matherly EE, Smith JN, Heistad DD, Anseth KS (2014) The role of valvular endothelial cell paracrine signaling and matrix elasticity on valvular interstitial cell activation. Biomaterials 35 : 3596-3606.

39. Frith JE, Menzies DJ, Cameron AR, Ghosh P, Whitehead DL, et al. (2014) Effects of bound versus soluble pentosan polysulphate in PEG/HA-based hydrogels tailored for intervertebral disc regeneration. Biomaterials 35: 1150 1162.

40. Farooque TM, Camp CH Jr, Tison CK, Kumar G, Parekh SH, et al. (2014) Measuring stem cell dimensionality in tissue scaffolds. Biomaterials 35: 25582567.

41. Daniele MA, Adams AA, Naciri J, North SH, Ligler FS (2014) Interpenetrating networks based on gelatin methacrylamide and PEG formed using concurren thiol click chemistries for hydrogel tissue engineering scaffolds. Biomaterials 35: $1845-1856$.

42. Dang LT, Feric NT, Laschinger C, Chang WY, Zhang B, et al. (2014) Inhibition of apoptosis in human induced pluripotent stem cells during expansion in a defined culture using angiopoietin-1 derived peptide QHREDGS. Biomaterials 35: 7786-7799.

43. Tokuda EY, Leight JL, Anseth KS (2014) Modulation of matrix elasticity with PEG hydrogels to study melanoma drug responsiveness. Biomaterials 35 4310-4318.

44. Nguyen MK, Jeon O, Krebs MD, Schapira D, Alsberg E (2014) Sustained localized presentation of RNA interfering molecules from in situ forming hydrogels to guide stem cell osteogenic differentiation. Biomaterials 35: 62786286

45. Lin TY, Ki CS, Lin CC (2014) Manipulating hepatocellular carcinoma cell fate in orthogonally cross-linked hydrogels. Biomaterials 35: 6898-6906.

46. Jeong CG, Francisco AT, Niu Z, Mancino RL, Craig SL, et al. (2014) Screening of hyaluronic acid-poly(ethylene glycol) composite hydrogels to support intervertebral disc cell biosynthesis using artificial neural network analysis. Acta Biomater 10: 3421-3430

47. Jeon O, Samorezov JE, Alsberg E (2014) Single and dual crosslinked oxidized methacrylated alginate/PEG hydrogels for bioadhesive applications. Acta Biomater 10: 47-55.

48. Hoffman MD, Van Hove AH, Benoit DS (2014) Degradable hydrogels for spatiotemporal control of mesenchymal stem cells localized at decellularized bone allografts. Acta Biomater 10: 3431-3441.

49. Zhang Z, Loebus A, de Vicente G, Ren F, Arafeh M, et al. (2014) Synthesis of Poly(ethylene glycol)-based Hydrogels via Amine-Michael Type Addition with Tunable Stiffness and Postgelation Chemical Functionality. Chem Mater 26: 3624-3630.

50. Xu J, Feng E, Song J (2014) Bioorthogonally Cross-Linked Hydrogel Network with Precisely Controlled Disintegration Time over a Broad Range. J Am Chem Soc 136: 4105-4108.

51. Wieduwild R, Lin W, Boden A, Kretschmer K, Zhang Y (2014) A repertoire of peptide tags for controlled drug release from injectable noncovalent hydrogel. Biomacromolecules 15: 2058-2066

52. McKinnon DD, Brown TE, Kyburz KA, Kiyotake E, Anseth KS (2014) Design and characterization of a synthetically accessible, photodegradable hydroge for user-directed formation of neural networks. Biomacromolecules 15: 28082816. 
Citation: Hutanu D, Frishberg MD, Guo L, Darie CC (2014) Recent Applications of Polyethylene Glycols (PEGs) and PEG Derivatives. Mod Chem appl 2: 132. doi:10.4172/2329-6798.1000132

53. McKinnon DD, Domaille DW, Cha JN, Anseth KS (2014) Bis-Aliphatic Hydrazone-Linked Hydrogels Form Most Rapidly at Physiological pH: Identifying the Origin of Hydrogel Properties with Small Molecule Kinetic Studies. Chem Mater 26: 2382-2387.

54. Ma Y, Fu X, Shen Y, Fu W, Li Z (2014) Irreversible Low Critical Solution Temperature Behaviors of Thermal-responsive OEGylated Poly(l-cysteine) Containing Disulfide Bonds. Macromolecules 47: 4684-4689.

55. Cencer M, Liu Y, Winter A, Murley M, Meng H, et al. (2014) Effect of pH on the Rate of Curing and Bioadhesive Properties of Dopamine Functionalized Poly(ethylene glycol) Hydrogels. Biomacromolecules 15: 2861-2869.

56. Azagarsamy MA, McKinnon DD, Alge DL, Anseth KS (2014) Coumarin-Based Photodegradable Hydrogel: Design, Synthesis, Gelation, and Degradation Kinetics. ACS Macro Lett 3: 515-519.

57. Kim KS, Park W, Hu J, Bae YH, Na K (2014) A cancer-recognizable MR contrast agents using $\mathrm{pH}$-responsive polymeric micelle. Biomaterials 35 : 337 343.

58. Song W, Tang Z, Zhang D, Zhang Y, Yu H, et al. (2014) Anti-tumor efficacy of c(RGDfK)-decorated polypeptide-based micelles co-loaded with docetaxel and cisplatin. Biomaterials 35: 3005-3014.
59. Mei L, Fu L, Shi K, Zhang Q, Liu Y, et al. (2014) Increased tumor targeted delivery using a multistage liposome system functionalized with RGD, TAT and cleavable PEG. Int J Pharm 468: 26-38.

60. Kong X, Yu K, Yu M, Feng Y, Wang J, et al. (2014) A novel multifunctiona poly(amidoamine) dendrimeric delivery system with superior encapsulation capacity for targeted delivery of the chemotherapy drug 10-hydroxycamptothecin Int J Pharm 465: 378-387.

61. Guo M, Mao H, Li Y, Zhu A, He H, et al. (2014) Dual imaging-guided photothermal/photodynamic therapy using micelles. Biomaterials 35: 46564666 .

62. Xue X, Ji S, Mu Q, Hu T (2014) Heat treatment increases the bioactivity of C-terminally PEGylated staphylokinase. Process Biochemistry 49: 1092-1096.

63. Xu P, Yu H, Zhang Z, Meng Q, Sun H, et al. (2014) Hydrogen-bonded and reduction-responsive micelles loading atorvastatin for therapy of breast cancer metastasis. Biomaterials 35: 7574-7587.

64. Kang T, Gao X, Hu Q, Jiang D, Feng X, et al. (2014) iNGR-modified PEGPLGA nanoparticles that recognize tumor vasculature and penetrate gliomas. Biomaterials 35: 4319-4332.

65. Bai J, Liu Y, Jiang X (2014) Multifunctional PEG-GO/CuS nanocomposites for near-infrared chemo-photothermal therapy. Biomaterials 35: 5805-5813. 